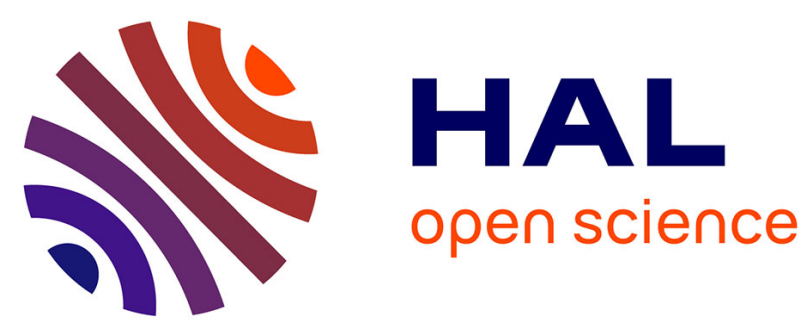

\title{
Improving magneto-inertial attitude and position estimation by means of magnetic heading observer
}

Charles-Ivan Chesneau, Mathieu Hillion, Jean-Francois Hullo, Guillaume Thibault, Christophe Prieur

\section{- To cite this version:}

Charles-Ivan Chesneau, Mathieu Hillion, Jean-Francois Hullo, Guillaume Thibault, Christophe Prieur. Improving magneto-inertial attitude and position estimation by means of magnetic heading observer. IPIN 2017 - 8th International Conference on Indoor Positioning and Indoor Navigation, Sep 2017, Sapporo, Japan. 10.1109/IPIN.2017.8115862 . hal-01670637

\section{HAL Id: hal-01670637 https://hal.science/hal-01670637}

Submitted on 5 Jan 2018

HAL is a multi-disciplinary open access archive for the deposit and dissemination of scientific research documents, whether they are published or not. The documents may come from teaching and research institutions in France or abroad, or from public or private research centers.
L'archive ouverte pluridisciplinaire HAL, est destinée au dépôt et à la diffusion de documents scientifiques de niveau recherche, publiés ou non, émanant des établissements d'enseignement et de recherche français ou étrangers, des laboratoires publics ou privés. 


\title{
Improving magneto-inertial attitude and position estimation by means of magnetic heading observer
}

\author{
Charles-Ivan Chesneau ${ }^{1,2}$, Mathieu Hillion ${ }^{1}$, Jean-François Hullo ${ }^{3}$, Guillaume Thibault ${ }^{4}$, Christophe Prieur ${ }^{2}$, \\ ${ }^{1}$ Sysnav \\ 57 Rue de Montigny, \\ 27200 Vernon, France \\ charles-ivan. chesneaulsysnav.fr \\ ${ }^{3}$ EDF Energy R\&D, \\ 40 Grosvenor Pl, Belgravia, \\ London SW1X 7EN, United Kingdom \\ ${ }^{2}$ Univ. Grenoble Alpes, \\ CNRS, GIPSA-lab, \\ F-38000 Grenoble, France \\ christophe.prieuregipsa-lab.fr \\ ${ }^{4} \mathrm{EDF}$ R\&D, \\ 7 boulevard Gaspard Monge, \\ 91120 Palaiseau, France
}

\begin{abstract}
This paper studies heading estimation jointly with the attitude and position estimation of a rigid body equipped with inertial and magnetic sensors in indoor environment. In contrast with other indoor dead-reckoning approaches, no assumption is made about the nature of the movement or environment layout. Based on a previous paper, an Extended Kalman Filter is designed, which includes inertial sensor biases and magnetic disturbances. A heuristic model of the dynamic of magnetic heading disturbances is then described and added to the observer. The latter is then evaluated in terms of position and heading error on experimental data, showing that in spite of high levels of disturbances, the magnetic field alone can be used to compute heading.
\end{abstract}

\section{INTRODUCTION}

Heading error is a major source of positioning error in indoor pedestrian dead-reckoning using low-cost MEMS sensors. Various solutions to mitigate such errors have been proposed, relying on the magnetic field [1], [2], and/or using assumptions about the shape of the building [3], [4], [5], [6]. A well-known issue is the high magnitude of magnetic disturbances in indoor environment, which is a big argument for ignoring it entirely as a source of heading information. In buildings whose corridors are aligned along dominant directions, Heuristic Drift Elimination (HDE) and its variations have been proved to be a reliable alternative.

The starting point of this paper is an Extended Kalman Filter $(\mathrm{EKF})$ observer estimating the attitude and displacement using magneto-inertial measurements, exploiting variations of the magnetic field with respect to time and space to indirectly estimate velocity [7]. In the model described therein, it can be shown that heading is not observable. Moreover, gyrometer and accelerometer biases are not modeled, leading to unreliable error estimates. Modifications are then necessary in order to keep heading errors from increasing, and improve error estimates.

First, a physics-driven model is provided in Section I. Then, it is augmented by a data-driven model to account for gyrometer and accelerometer biases, and unexpected magnetic field variations, from which an EKF can be de- signed in Section II. A simple model is then proposed to help reject magnetic disturbances in heading estimation, and the subsequent modifications of the EKF observer are described in Section III. Model dynamics involved in heading estimation are dynamically tuned depending on the magnetic gradient and the estimated velocity. This choice is justified from empirical observations made in SYSNAV's office. Performance is then evaluated from experimental data. Experimental results show a clear improvement in estimation accuracy on long walks, in comparison with [7] (see Sections IV and V for experiments in a public building and in an industrial facility respectively).

\section{Problem Statement}

The problem considered is the motion tracking of a rigid sensor board using strapdown MEMS inertial and magnetic sensors. The kinematic model is described in Section I-A, and measurements in Section I-B. The problem is then to find an observer recursively computing attitude, heading, position and error estimates in a dead-reckoning framework, aiming at minimizing drift in position estimates.

\section{A. Continuous-time kinematic model}

Let $\mathfrak{R}_{\mathrm{i}}$ be an inertial frame of reference and $\mathfrak{R}_{\mathrm{b}}$ a frame of reference moving with the rigid body. Coordinates of vectors in $\mathfrak{R}_{\mathrm{i}}$ (resp. $\mathfrak{R}_{\mathrm{b}}$ ) are denoted with the prescript ${ }^{\mathrm{i}}$ (resp. ${ }^{\mathrm{b}}$ ). Let $\mathbf{P}$ denote the accelerometer point of percussion, which is a fixed point in $\Re_{\mathrm{b}}$. Let $\mathbf{v}$ be the velocity vector of $\mathbf{P}$ in $\mathfrak{R}_{\mathrm{i}}$, and a the associated acceleration vector. Then, by definition,

$$
\begin{aligned}
& \mathbf{v}(t) \triangleq \frac{\mathrm{d} \mathbf{P}(t)}{\mathrm{d} t} \\
& \mathbf{a}(t) \triangleq \frac{\mathrm{d} \mathbf{v}(t)}{\mathrm{d} t} .
\end{aligned}
$$

The cinematic acceleration vector a can be decomposed into the gravitational acceleration $\mathbf{g}$, and the proper acceleration $\gamma(t)$, so that:

$$
\mathbf{a}(t)=\gamma(t)+\mathbf{g}
$$




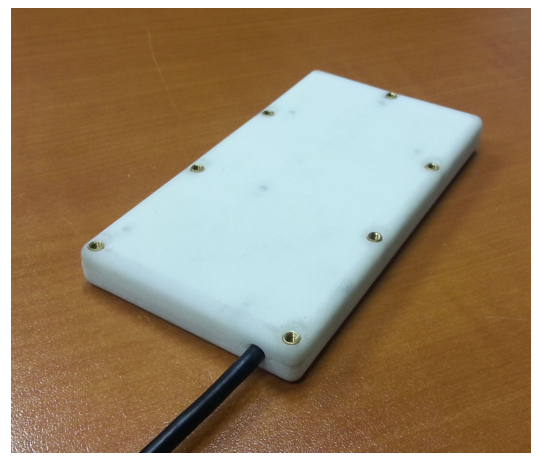

Figure 1. Picture of the sensor board package

Let $\mathbf{M}$ (may be different from $\mathbf{P}$ ) denote the fixed point of the rigid body at which the magnetic field $\mathbf{B}$ is assumed to be measured:

$$
\frac{\mathrm{d} \mathbf{B}(\mathbf{M}(t), t)}{\mathrm{d} t}=\nabla \mathbf{B}(t) \frac{\mathrm{d} \mathbf{M}(t)}{\mathrm{d} t}+\left.\frac{\partial \mathbf{B}(x, t)}{\partial t}\right|_{(\mathbf{M}(t), t)}
$$

where:

$$
\left.\nabla \mathbf{B}(t) \triangleq \frac{\partial \mathbf{B}(x, t)}{\partial x}\right|_{(\mathbf{M}(t), t)} .
$$

Assuming two orthonormal basis are respectively given for $\mathfrak{R}_{i}$ and $\mathfrak{R}_{b}$, and neglecting the angular rate of $\mathfrak{R}_{i}$, the attitude matrix $R$ can be defined such that:

$$
\frac{\mathrm{d} R(t)}{\mathrm{d} t}=R(t)\left[{ }^{\mathrm{b}} \omega \times\right]
$$

where $\boldsymbol{\omega}$, whose body frame coordinates are $\left({ }^{\mathrm{b}} \omega_{1},{ }^{\mathrm{b}} \omega_{2},{ }^{\mathrm{b}} \omega_{3}\right)^{\top}$, is the angular rate vector of the body frame, and:

$$
\left[{ }^{\mathrm{b}} \omega \times\right] \triangleq\left(\begin{array}{ccc}
0 & -{ }^{\mathrm{b}} \omega_{3} & { }^{\mathrm{b}} \omega_{2} \\
\mathrm{~b}^{\mathrm{b}} \omega_{3} & 0 & -{ }^{\mathrm{b}} \omega_{1} \\
-{ }^{\mathrm{b}} \omega_{2} & { }^{\mathrm{b}} \omega_{1} & 0
\end{array}\right) .
$$

Assuming either $\left.\frac{\partial \mathbf{B}(x, t)}{\partial t}\right|_{(\mathbf{M}(t), t)}=0$ or that the origin of $\left.\frac{\partial \mathbf{B}(x, t)}{\partial t}\right|_{(\mathbf{M}(t), t)}$ be only power-line interference has lead to the design of observers that were successful in delivering velocity estimates in practice [8], [7]. However, attempting to account for sensor biases with such models seems to cause robustness issues. In fact, while power-line interference in most indoor environments is a strong and ubiquitous source of disturbance, people carrying metallic objects or magnets, moving furniture such as office chairs, nearby passing trains, or direct current power supplies can all cause measurable instationarities in the magnetic field outside of power-line interference frequencies.

\section{B. Hardware description and model of available measure- ments}

A smartphone-sized sensor board (Fig. 1) provides three types of measurements sampled by $\Sigma-\Delta$ analog to digital converters at a frequency $1 / T$, with $T=3.072 \mathrm{~ms}$ : accelerometer, gyrometer, and magnetometer measurements from a magnetometer array. The latter are processed in order to provide an estimation of the magnetic field $\hat{B}[k]$ and its spatial gradient $\nabla \hat{B}[k]$ in body frame. Sampling can be modeled as a weighted integration or convolution. Let us denote $m_{\mathrm{IMU}}$ and $m_{\mathrm{MAG}}$ two convolution functions. Let us denote $\hat{\omega}[k]$ and $\hat{\gamma}[k]$ sampled measurements of strapdown gyrometers and accelerometers at time-step $k$ such that:

$$
\begin{array}{r}
\hat{\omega}[k]-b_{\omega}[k]+\nu_{\omega}[k]=\left(m_{\mathrm{IMU}} \star{ }^{\mathrm{b}} \omega\right)(k T) \\
\hat{\gamma}[k]-b_{\gamma}[k]+\nu_{\gamma}[k]=\left(m_{\mathrm{IMU}} \star^{\mathrm{b}} \gamma\right)(k T) \\
\hat{B}[k]+\nu_{B}[k]=\left(m_{\mathrm{MAG}} \star^{\mathrm{b}} B\right)(k T) \\
\nabla \hat{B}[k]+\nu_{\nabla B}[k]=\left(m_{\mathrm{MAG}}{ }^{\mathrm{b}} \nabla B\right)(k T)
\end{array}
$$

where $b_{\gamma}$ and $b_{\omega}$ denote respectively the coordinates in body frame of accelerometer and gyrometer biases at time step $k$, $\nu_{\omega}, \nu_{\gamma}, \nu_{B}[k], \nu_{\nabla B}[k]$ denote respectively white noise zero mean gaussian processes with known variances.

\section{Observability issue}

Let us consider a change of reference frame $\mathfrak{R}_{\mathrm{i}}$ which is a rotation around the vertical axis defined by $\mathrm{g}$, and a change of origin. Then model description is invariant with respect to this change of reference frame, which means that both heading and the position are unobservable. Their estimates are then defined in an arbitrary reference frame and subject to drift.

Section II aims at minimizing heading drift by first modeling estimation error accounting for inertial sensor biases and magnetic disturbances. The underlying model is then augmented in order to solve the heading unobservability problem in Section III.

\section{EKF OBSERVER}

For practical reasons, a discrete-time extended Kalman filter is chosen to address the observation problem. Therefore, a discrete-time dynamic model and measurement model must be provided. It is obtained by discretization of a continous-time model described in Sections II-A and II-B. Error variables used to describe the state-space are described in Section II-C. The EKF is then computed on the error state, in a way described in $[9$, ch.15].

\section{A. Continuous-time dynamic model}

The continuous-time dynamic model used to compute the EKF is divided into three parts, the kinematics laws:

$$
\left\{\begin{aligned}
\dot{R} & =R[\omega \times] \\
{ }^{\mathrm{i}} \dot{P} & =R^{\mathrm{b}} v \\
{ }^{\mathrm{b}} \dot{v} & =-[\omega \times]^{\mathrm{b}} v+\gamma+R^{\top \mathrm{i}} g \\
{ }^{\mathrm{b}} \dot{B} & =-[\omega \times]^{\mathrm{b}} \dot{B}+{ }^{\mathrm{b}} \nabla B^{\mathrm{b}} v+R^{\top} B^{(\mathrm{p})},
\end{aligned}\right.
$$

a model of magnetic disturbances:

$$
\left\{\begin{array}{l}
{ }^{\mathrm{i}} \dot{B}^{(\mathrm{p})} \approx-\frac{{ }^{\mathrm{i}} B^{(\mathrm{p})}}{\tau_{B^{(\mathrm{p})}}} \\
{ }^{\mathrm{i}} \ddot{B}^{(\mathrm{pl})} \approx-\omega^{(\mathrm{pl})} \omega^{(\mathrm{pl})}{ }^{\mathrm{i}} B^{(\mathrm{pl})}
\end{array}\right.
$$


and a model of sensor biases dynamics:

$$
\left\{\begin{array}{l}
\dot{b}_{\omega} \approx-\frac{b_{\omega}}{\tau_{b_{\omega}}} \\
\dot{b}_{\gamma} \approx-\frac{b_{\gamma}}{\tau_{b_{\gamma}}} .
\end{array}\right.
$$

Here $\mathbf{B}^{(\mathrm{pl})}$ denotes the magnetic field induced by powerline interference, assumed space-invariant in $\mathfrak{R}_{\mathrm{i}}$ oscillating at frequency $\frac{\omega^{(\mathrm{pl})}}{2 \pi}$. These hypotheses allow reliable rejection of this dynamic in indoor spaces or urban environment. The actual discrete-time model for power-line interference is written as in [10].

\section{B. Measurement model}

The only measurement considered is the magnetic field, affected by power-line interference:

$$
y_{B}={ }^{\mathrm{b}} B+R^{\top \mathrm{i}} B^{(\mathrm{pl})}
$$

\section{Error state}

Let $\tilde{R}$ represent the attitude error, such that if $R$ represents the actual attitude and $\hat{R}$ the current estimate, it holds:

$$
R=\tilde{R} \hat{R} \text {. }
$$

The attitude error $\tilde{R}$ can be represented instead by its corresponding value in the tangent space in $\mathrm{SO}_{3} \tilde{\theta}$, related to $\tilde{R}$ by matrix exponentiation: $\tilde{R} \triangleq \exp [\tilde{\theta} \times] \cdot{ }^{1}$ The following error state vector is used:

$$
\tilde{x}=\left(\tilde{b}_{\omega}, \tilde{b}_{\gamma}, \tilde{\theta},{ }^{\mathrm{b}} \tilde{v},{ }^{\mathrm{i}} \tilde{B}_{\mathrm{p}},{ }^{\mathrm{b}} \tilde{B},{ }^{\mathrm{i}} \tilde{B}^{(\mathrm{pl})},{ }^{\mathrm{i}} \tilde{P}\right)^{\top},
$$

\section{HEADING ESTIMATION}

\section{A. Recursive magnetic field-based heading estimation}

Pure heading estimation with a Kalman filter is a rather straightforward problem to model. In discrete-time, this leads to the one-state, one measurement model of the form:

$$
\begin{aligned}
\psi[k+1] & =\psi[k]+\Delta \psi[k] \\
z_{\psi} & =\psi+\nu_{z_{\psi}}
\end{aligned}
$$

where $\psi[k]$ represents the heading at time-step $k, \Delta \psi[k] \triangleq$ $\psi[k+1]-\psi[k]$ its variation between time-steps $k$ and $k+1$ that can be typically estimated from gyrometer measurements, and $z_{\psi}$ a noisy measurement of heading, using the horizontal projection of the magnetic field as a heading reference.

In our case, heading measurement is affected by a noise that is typically not white and Gaussian. Illustrating the issue, a first try at modeling disturbance dynamics leads to:

$$
\psi^{(\mathrm{d})}[k+1]=\psi^{(\mathrm{d})}[k]+u[k] .
$$

assuming an approximate value of $u$ can be provided, with a redefinition of the heading measurement:

$$
z_{\psi}=\psi+\psi^{(\mathrm{d})}+\nu_{z_{\psi}}
$$

\footnotetext{
${ }^{1}$ It was verified in a motion capture experiment that was not included in this paper that $\tilde{R}$ stays close to identity, meaning that the attitude error stays small.
}

where $\psi^{(\mathrm{d})}$ stands for heading measurement noise. Because $u$ is the variation of the magnetic heading disturbance, its value can be predicted in the context of [7]. However, this model is still unobservable, since the argument in Section I-C still holds.

Moreover, if one wanted to use the Kalman filter as its recursive Bayesian estimator interpretation, one could remark from a few counter-examples that using the EKF with a small fixed number of states is not the appropriate tool. For example, for any periodic movement, one would fail to model correlations between magnetic disturbances from each loop. This is because the disturbance depends on position and not on time (or time-step), breaking the Markovian property of the underlying model.

Despite this, for computational reasons, we chose to use the EKF. Another Markovian model for $\psi^{(\mathrm{d})}$ is then needed in order to proceed.

\section{B. Model with disturbances}

Let us find a recursive model for the dynamics of $\psi^{(\mathrm{d})}$ that captures:

- Its variations between one time-step and the next

- Prior information about the magnitude of the local magnetic disturbance

To that end, let us define the joint prior probability distribution:

$$
p\left(\left(\begin{array}{c}
\psi^{(\mathrm{d})}[k] \\
\psi^{(\mathrm{d})}[k+1]
\end{array}\right)\right) \sim \mathcal{N}\left(\left(\begin{array}{c}
\mu[k] \\
\mu[k+1]
\end{array}\right), \Sigma[k]\right)
$$

where the covariance matrix can be written as $\Sigma[k] \triangleq$

$\left(\begin{array}{cc}a[k]^{2} & \alpha[k] a[k] b[k] \\ \alpha[k] a[k] b[k] \quad & b[k]^{2}\end{array}\right)$,
$-1<\alpha[k]<1$.

Let us assume that $\hat{u}[k]$ is known such that (26) implies:

$$
\psi^{(\mathrm{d})}[k+1]-\psi^{(\mathrm{d})}[k]=u[k] \sim \mathcal{N}\left(\hat{u}[k], \sigma_{u}[k]^{2}\right) .
$$

Then,

$$
\begin{aligned}
\mu[k+1]-\mu[k] & =\hat{u}[k] \\
\left(\begin{array}{ll}
-1 & 1
\end{array}\right) \Sigma[k]\left(\begin{array}{c}
-1 \\
1
\end{array}\right) & =\sigma_{u}[k]^{2},
\end{aligned}
$$

which reads $a[k]^{2}+b[k]^{2}-2 a[k] b[k] \alpha[k]=\sigma_{u}[k]^{2}$. Neglecting $|a[k]-b[k]|$ before $|a[k]|$, where $a[k]$ represents the a priori local disturbance standard deviation at time-step $k$ :

$$
\alpha[k]=1-\frac{\sigma_{u}[k]^{2}}{2 a[k]^{2}} .
$$

From this assumption, the following conditional probability is obtained:

$$
\begin{array}{r}
p\left(\psi^{(\mathrm{d})}[k+1] \mid \psi^{(\mathrm{d})}[k]\right) \sim \mathcal{N}\left(\alpha[k]\left(\psi^{(\mathrm{d})}+\hat{u}[k]\right)\right. \\
+(1-\alpha[k]) \mu[k+1] \\
\left.a[k]^{2}\left(1-\alpha[k]^{2}\right)\right)
\end{array}
$$


The following equation is then used to predict the disturbance in discrete-time:

$$
\begin{aligned}
\psi^{(\mathrm{d})}[k+1]=\alpha[k]\left(\psi^{(\mathrm{d})}\right. & +\hat{u}[k]) \\
& +(1-\alpha[k]) \frac{\hat{u}[k]}{2}+\nu_{\psi^{(\mathrm{d})}}[k]
\end{aligned}
$$

where $\nu_{\psi(\mathrm{d})}[k]$ is a Gaussian variable of variance $a[k]^{2}(1-$ $\left.\alpha[k]^{2}\right)$.

The discrete-time dynamic model given by (22) and (29) with the measurement model (25) is an observable model that can then be used in a Kalman filter. The obtained inputoutput relationship from $z_{\psi}$ to $\psi^{(\mathrm{d})}$ is essentially a highpass filter with a feed-forward term. In the following, $b[k]$ is chosen equal to $a[k]$. Choices of $a[k]$ and $\sigma_{u}[k]$ are then discussed in Sections III-C and III-D.

\section{Heading estimation in the full $3 D$ problem}

In the full 3-D problem, we propose adding two variables to the state vector, and one scalar measurement. To help using multivariate Gaussian distributions to represent uncertainties, attitude and trajectory are then represented in an inertial frame with arbitrary heading, and an additional scalar variable $\tilde{\psi}^{\text {(init) }}$ is added to the error state in order to represent the arbitrary initial heading. Since $\psi^{(\text {init })}$ is constant by definition, we have:

$$
\forall k, \psi^{(\text {init) }}[k+1]=\psi^{(\text {init })}[k] .
$$

With $\tilde{\psi}^{(\mathrm{d})}$ also added to error state, equations (29) and (30) are then combined to get the full discrete-time model.

Let $\mathfrak{R}_{\mathrm{m}}$ be a frame of reference whose north is defined by the geomagnetic field. Let us define $\psi^{\text {(init) }}$ such that

$$
\begin{aligned}
R^{(\text {init })} & \triangleq\left(\begin{array}{ccc}
\cos \psi^{(\text {init })} & -\sin \psi^{(\text {init })} & 0 \\
-\sin \psi^{(\text {init })} & \cos \psi^{(\text {init })} & 0 \\
0 & 0 & 1
\end{array}\right) \\
{ }^{\mathrm{i}} g_{1} & =0 \\
{ }^{\mathrm{i}} g_{2} & =0 \\
{ }^{\mathrm{m}} B^{(\mathrm{t})} & \triangleq R^{(\text {init })}\left(R^{\mathrm{b}} B\right) \\
\psi^{(\mathrm{d})} & \triangleq \arctan \left(\frac{{ }^{\mathrm{m}} B_{1}^{(\mathrm{t})}}{{ }^{\mathrm{m}} B_{2}^{(\mathrm{t})}}\right) \triangleq \phi\left({ }^{\mathrm{m}} B^{(\mathrm{t})}\right)
\end{aligned}
$$

Let us denote:

$$
u[k]=\phi\left(R[k+1]^{\mathrm{b}} B[k+1]\right)-\phi\left(R[k]^{\mathrm{b}} B[k]\right)
$$

Then (35) can be used as a scalar measurement equation for Kalman filtering in the 3D problem, by substituting ${ }^{\mathrm{m}} B$ with its expression in (34), then substituting $R^{\mathrm{b}} B$ with a function of $y_{\mathrm{B}}$ and $B^{(\mathrm{pl})}$ by using (19).

Tuning parameters are computed using the following heuristic: $\sigma_{u}$ is chosen such that $\sigma_{u} \propto\|v\|$, and $a[k]$ models the expected magnitude of the local disturbance. It follows that the model reduces to the classic AHRS model when $a[k]=0$, and its asymptotic behavior when $\sigma_{u}$ is close to zero is comparable to the quasi-static field approach.

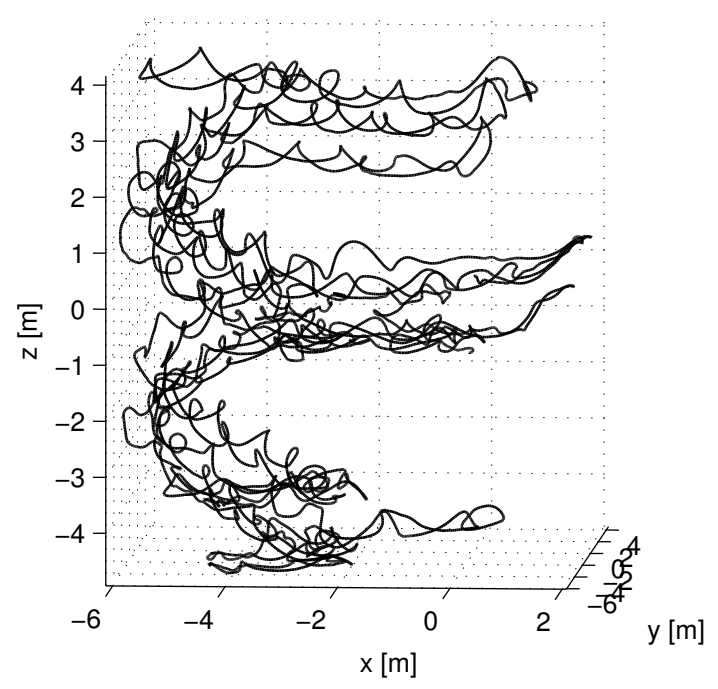

Figure 2. Reference multi-floor trajectory for magnetic heading model calibration

\section{The magnetic gradient as a variable of interest}

In practice, tuning $\sigma_{u}$ may lead to a static proportionality coefficient with respect to velocity. However, tuning $a[k]$ reveals slightly more problematic. A small value leads to a faster convergence but poor disturbance rejection, while a high value leads to poor convergence rates but good disturbance rejection with respect to magnetic disturbances. A heuristic would be useful in order to chose a pertinent tuning for $a[k]$ from measurements, that is to say, increasing with the expected amplitude of the local magnetic disturbance.

Among trajectories used in [7], one of them is chosen for its relatively small heading drift and big swept volume (Fig. 2). Magnetic declination can be computed for each time-step in the arbitrary inertial frame (Fig. 3). Data is then sorted by magnetic gradient norm, clustered, and the standard deviation is computed for each cluster (Fig. 4). One can notice that while the magnitude of magnetic disturbances remains rather high, its distribution matches noticeably well with an affine model with respect to the magnetic gradient. This suggests the following empirical model:

$$
a[k]=a_{0}+a_{1} N[k],
$$

where

$$
N[k]=\|\nabla \hat{B}[k]\|
$$

Other publications such as [1], [11] suggest to compare the magnetic norm and dip to a geomagnetic model. However, on the same data, these variables do not seem to provide a reliable prior about the magnitude of the local disturbance of the magnetic declination. Empirical data rather suggests that in general, the local declination, norm and dip of the magnetic field actually behave like independent variables. This observation justifies not using these properties to compute a value for $a[k]$. 


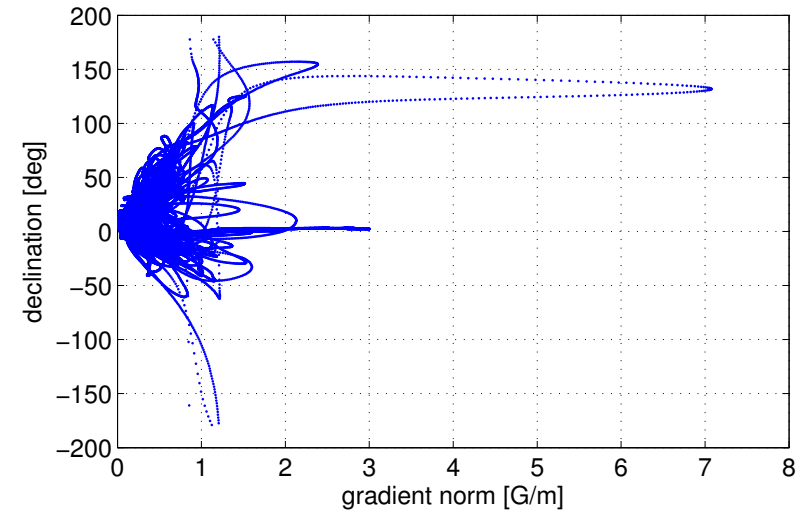

Figure 3. Estimated magnetic declination in inertial frame with respect to the gradient norm

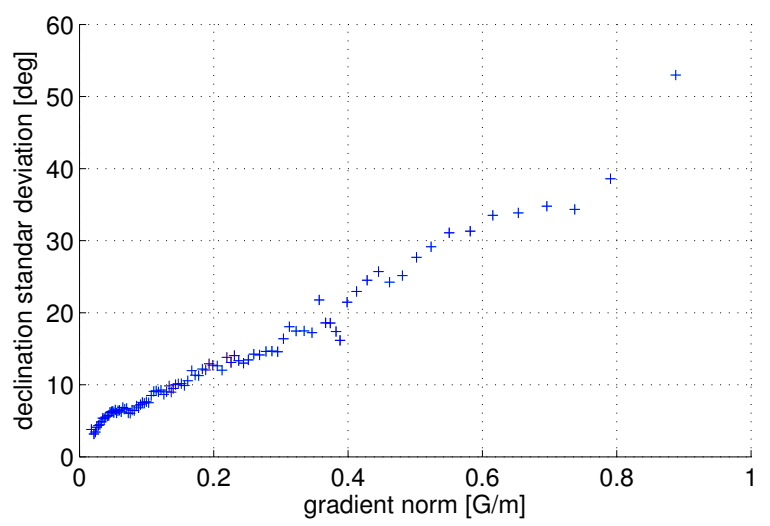

Figure 4. Declination standard deviation with respect to gradient norm

\section{EXPERIMENTAL RESULTS}

Two data sets are chosen in order to illustrate the effect of magnetic heading estimation on the trajectory accuracy. In each case, the sensor board is strapped on the user's trunk.

The first data set was acquired during an unofficial trial at the IPIN 2016 competition in Alcalá de Henares, Spain, on the actual path of the pedestrian dead reckoning competition. The path included 57 geo-referenced waypoints. The described observer is compared with the one published in [7] in the following manner: algorithms are started on the first waypoint with no initialization procedure beforehand. Since trajectories are computed in the same arbitrary initial reference frame, both are rotated by the final estimate of $\psi^{\text {(init) }}$ computed by the new algorithm, corrected by a magnetic declination of -0.93 degrees and displayed on the same map (see Fig. 5-8). The solid blue trajectory is computed with the new algorithm, the dotted green one is computed with the old algorithm, and solid red bars are plotted between waypoints and their corresponding estimate according to the new algorithm. A clear improvement in performance is observed (see Fig. 9-10) comparing the algorithm in [7] with the current one. The output heading of the old algorithm drifts clockwise, which seems to cause most of the error

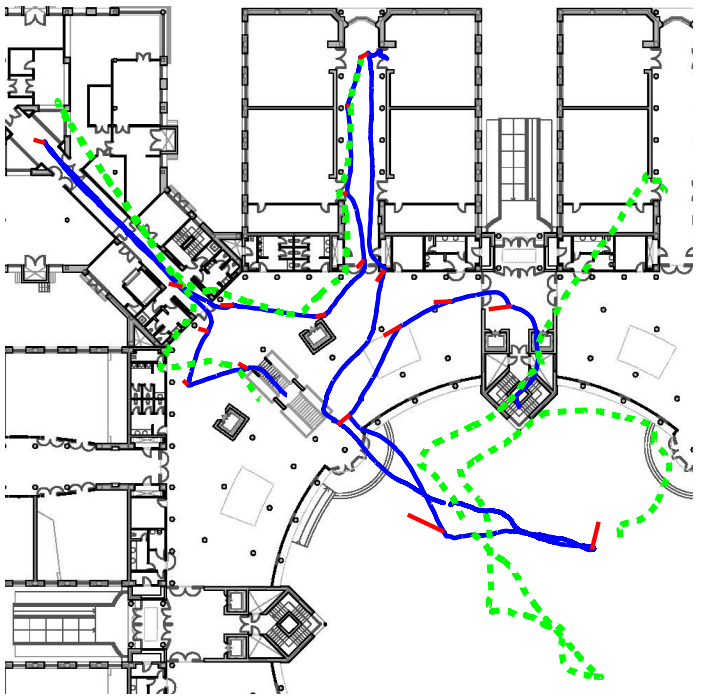

Figure 5. Computed trajectory on the path of IPIN 2016 Track 2, ground floor

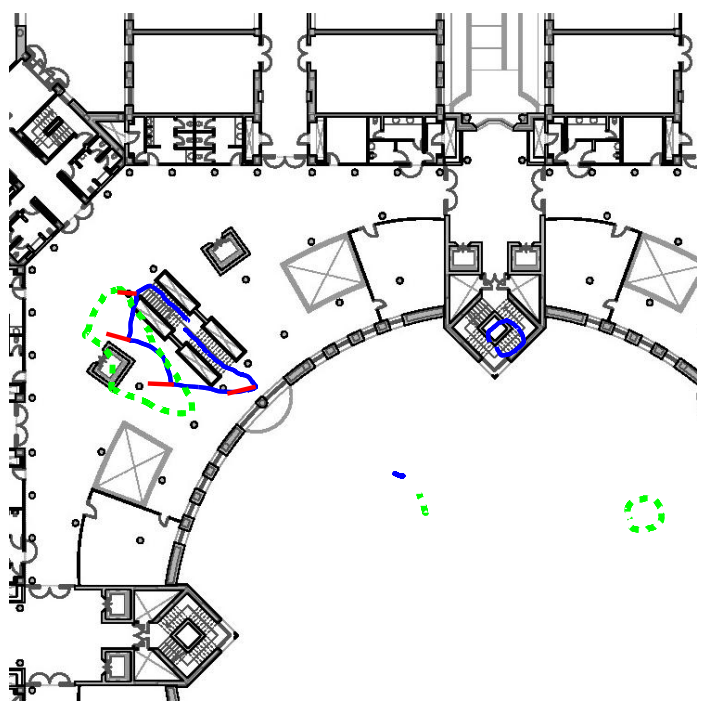

Figure 6. Computed trajectory on the path of IPIN 2016 Track 2, first floor

growth after waypoints number 20-25. The new algorithm, that includes magnetic heading estimation, does not display this behavior.

The second data set is acquired in a building with a heading reference. The heading reference consists in two references materializing a known geographical direction. After compensating for local declination according to a geomagnetic model, heading error is inferred from computing the traveling direction between the two references repeatedly (see Fig. 11). Movement is stopped during 10 minutes in front of a ferromagnetic structure, resulting in a strong magnetic disturbance. The maximum estimated 


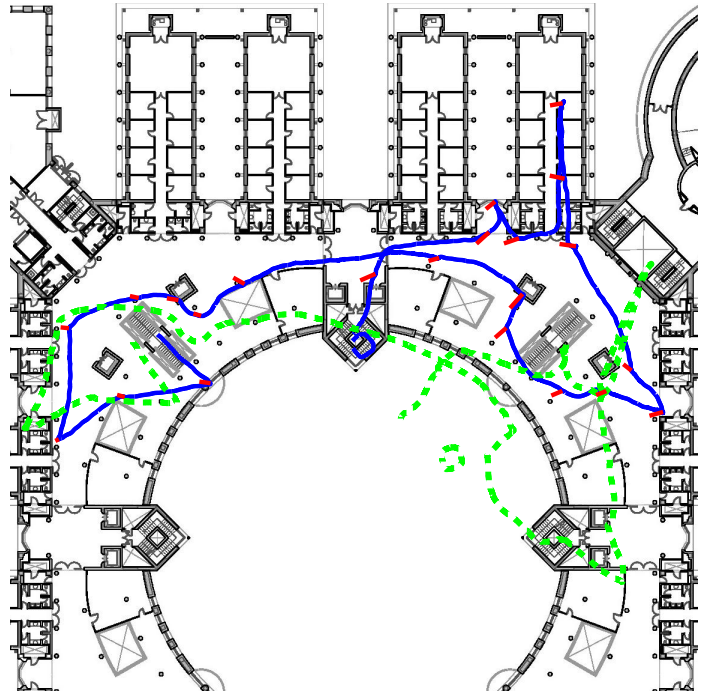

Figure 7. Computed trajectory on the path of IPIN 2016 Track 2, second floor

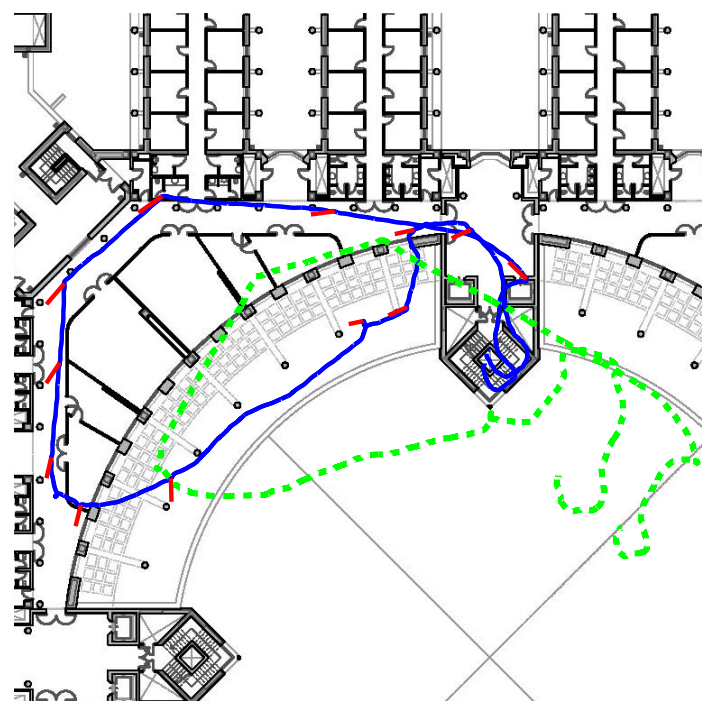

Figure 8. Computed trajectory on the path of IPIN 2016 Track 2, third floor

heading error immediately after initialization is 6.2 degrees, 4.8 degrees excluding the first measurement. The standard deviation of estimated heading error is 1.9 degrees.

\section{EXPERIMENT IN AN INDUSTRIAL FACILITY}

The magneto-inertial measurement unit has been tested in an industrial facility of Électricité de France (EDF). The facility extends over several floors, each containing a rather complex "labyrinth" of hallways, small chambers and platforms, which are connected to each other by many stairs and ladders. The building itself is a construction of mainly steel and reinforced concrete and thus represents also

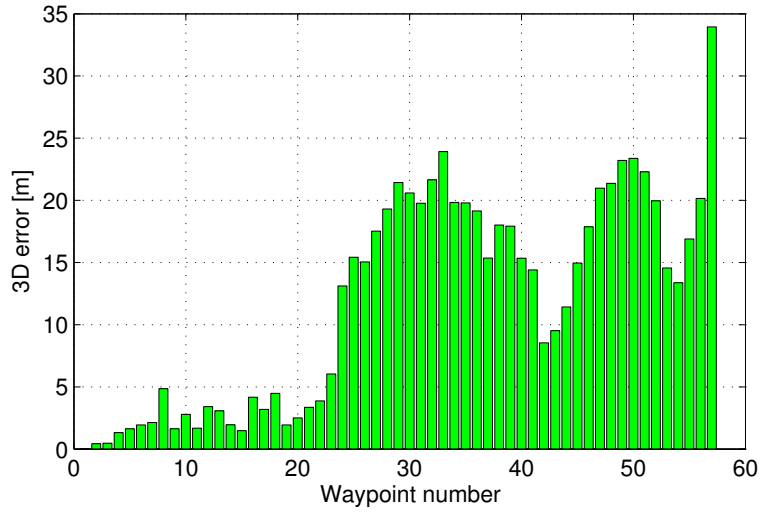

Figure 9. Distance from ground truth with the old algorithm. The third quartile of error is $19.76 \mathrm{~m}$

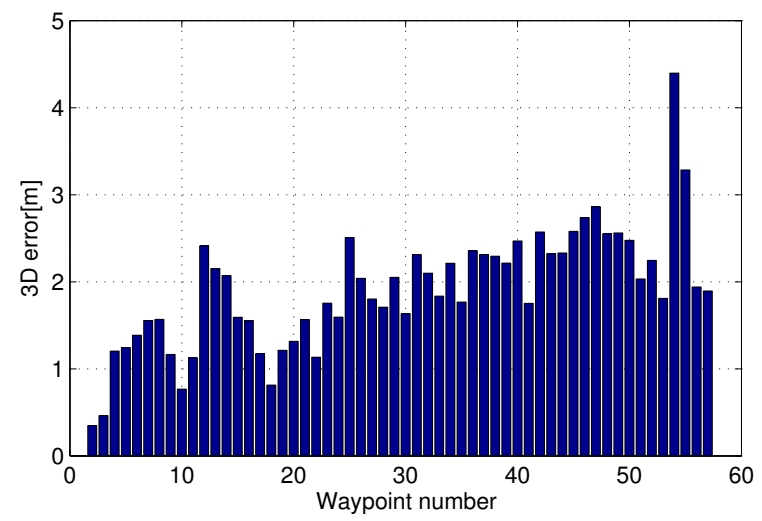

Figure 10. Distance from ground truth with the new algorithm. The third quartile of error is $2.32 \mathrm{~m}$

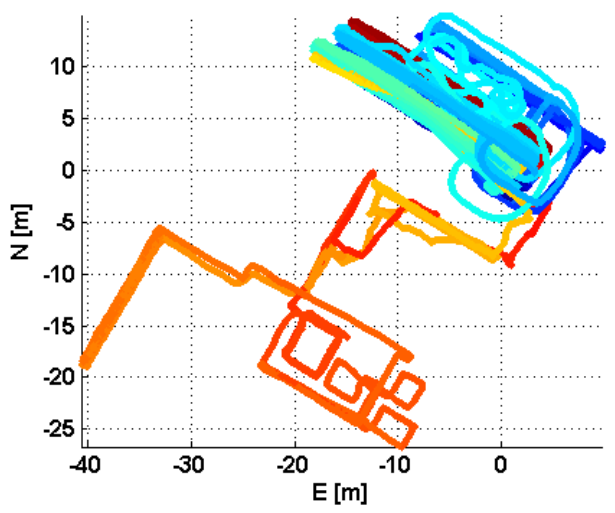

Figure 11 . A $2.5 \mathrm{~km}, 56 \mathrm{~min}$ long benchmark trajectory 


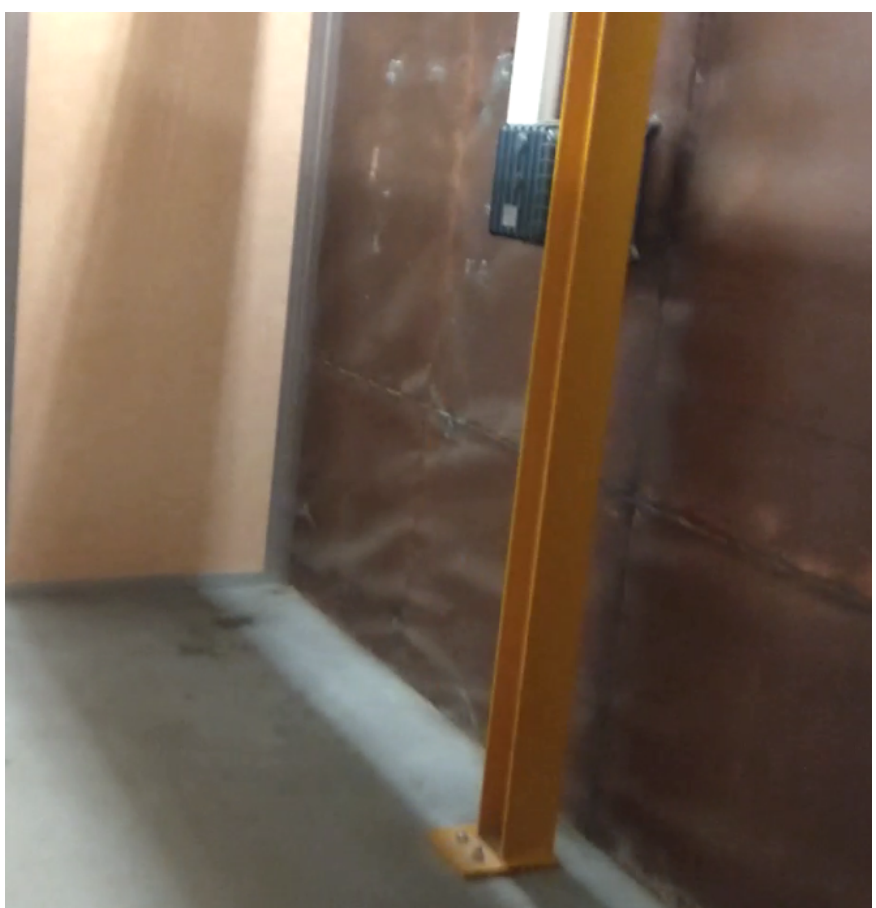

Figure 12. Beam in front of which the user is sitting for $10 \mathrm{~min}$ in the middle of the benchmark trajectory

magnetically a challenging environment for the measurement unit and the EKF. The main aspect of this building is that it is complex, with close to no dominant direction, and many ladders allowing changes between sectors on different levels. While magneto-inertial dead reckoning benefits from the relatively high level of magnetic gradient and indifference towards the type of movement, magnetic heading estimation is difficult.

Figures 13 to 15 show partial results of our analysis (see in particular the green lines in Figure 13 (a), (b) and in Figure 14). We have considered seven indoor trajectories, each over a time interval of 10 to 30 minutes. After a suitable initialization of the heading for each trajectory, the EKF output reproduced the trajectories as noted in a "logbook" during the course.

In the industrial facility under study in this section, an accurate map is available. It can be used with a map-matching technique combining the EKF output with a standard particle filter approach [12], [13], in which the random twodimensional motion of the particles on one floor is governed by the estimates that the EKF provides for the velocity and heading as well as their covariances. In our own " $2.5 \mathrm{D}$ " approach for indoor map-matching, we allow the particles to change levels by passing through sectors that represent stairs or ladders and connect floors of different height levels. While this idea bears a similarity with the three-dimensional particle filter as described in reference [14], we use the $z$ component only to detect when altitude is changing and, once it stabilizes again, to estimate the change in altitude during the period of change. This estimate allows us to
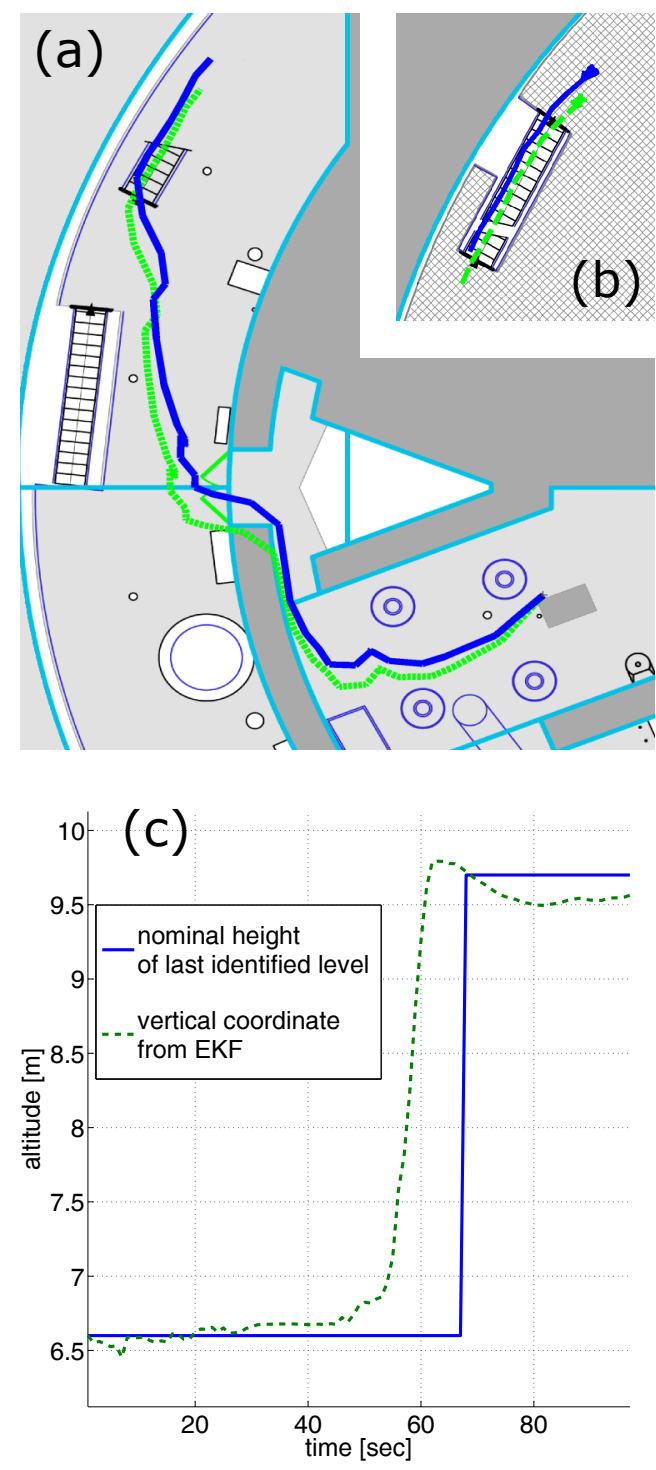

Figure 13. Experimental results for a complex industrial facility: (a) "Raw" trajectory obtained from the EKF (dashed green line) and the map-matched trajectory obtained by applying the particle filter on the EKF output (solid blue line). (b) is the continuation of (a) on a higher level of the facility. (c) shows the altitude predicted by the EKF (initialized at the beginning by the nominal height of the floor) as well as the nominal height of the floor that has last been recognized by the " $2.5 \mathrm{D}$ " map-matching algorithm.

identify transitions from one level to another, and eliminate all particles that randomly end up on the wrong floor. The results of this combination of the EKF output with the mapmatching approach is given in Figures 13 to 15 where, by comparing the green dashed lines (for the EKF output) and the blue solid lines (for the EKF output combined with the map-matching approach), the usefulness of the EKF output data for subsequent map-matching is shown in a challenging indoor environment, since it correctly reproduces complex trajectories over long distances. 


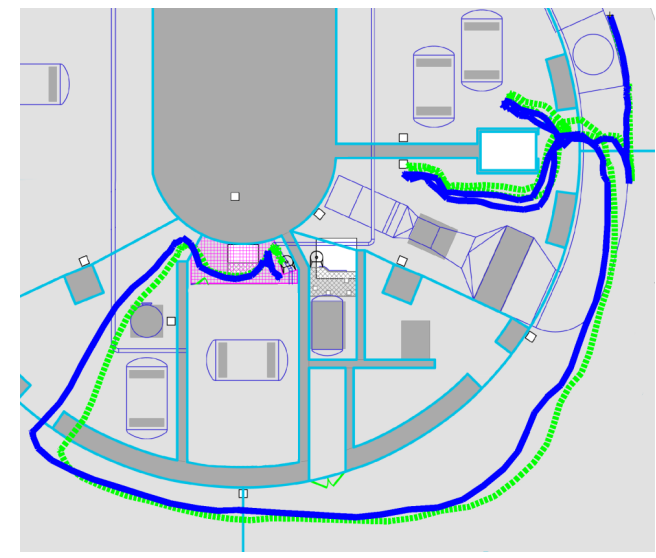

Figure 14. Trajectories estimated by the EKF (drashed green line) and the map-matching trajectory obtained by applying the particle filter on the EKF output (solid blue line).

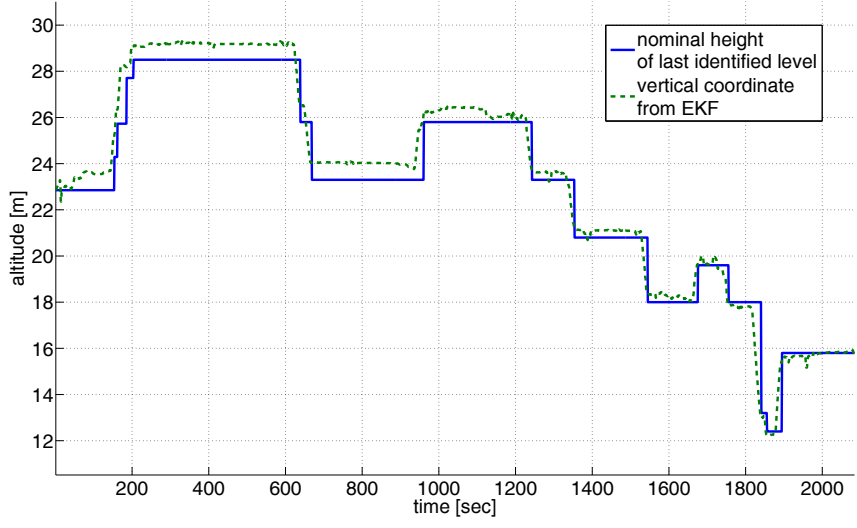

Figure 15. Altitude estimate of the magneto-inertial measurement unit (red curve) and the (nominal) altitude of the identified floor (blue curve) as a function of time for a course of 30 minutes through the labyrinth of the facility.

\section{CONCLUSion, Future Work}

This paper extends previous practical developments on magneto-inertial dead-reckoning. It shows how the magnetic field can be used indoor as a source of information both for heading and velocity estimation, without relying on a specific building shape. This approach prevents heading drifts during long walks and avoids hypothesis about the shape of the building. Preliminary results show that tuning the observer for each building was not necessary.

However, a more accurate model would be needed in order to take into account closed trajectories, through loop closure or models accounting for time invariant nature of most magnetic disturbances. Furthermore, research is needed in order to further increase robustness towards magnetic instationarity caused by moving ferromagnetic materials. Finally, some further studies may allow to improve the results obtained in Section V when combining an EKF with a particle filter and an accurate map.

\section{ACKNOWLEDGMENTS}

The experiments in the industrial facility and their analyses have been carried out within an R\&D contract with Électricité de France (EDF) dedicated to the advancement of high-precision indoor localisation. The authors would like to thank EDF for their support and the realization of the experiments in one of their facilities. The authors are grateful to EDF for the realization of the experiments in one of their facilities and would like to thank L. Le Tarnec and H. Meier of Sysnav for their assistance with the map-matching algorithm.

\section{REFERENCES}

[1] W. T. Faulkner, R. Alwood, W. T. David, and J. Bohlin, "Gps-denied pedestrian tracking in indoor environments using an imu and magnetic compass," in Proceedings of the 2010 International Technical Meeting of The Institute of Navigation, (San Diego, CA), pp. 198 - 204, January 2010.

[2] M. H. Afzal, V. Renaudin, and G. Lachapelle, "Use of earth's magnetic field for mitigating gyroscope errors regardless of magnetic perturbation," Sensors, vol. 11, no. 12, pp. 11390-11414, 2011.

[3] J. Borenstein and L. Ojeda, "Heuristic drift elimination for personnel tracking systems," Journal of Navigation, vol. 63, pp. 591-606, Sep 2010 .

[4] K. Abdulrahim, C. Hide, T. Moore, and C. Hill, "Aiding MEMS IMU with building heading for indoor pedestrian navigation," 2010 Ubiquitous Positioning Indoor Navigation and Location Based Service, Oct 2010.

[5] A. R. Jimenez, F. Seco, F. Zampella, J. C. Prieto, and J. Guevara, "Improved heuristic drift elimination (iHDE) for pedestrian navigation in complex buildings," in 2011 International Conference on Indoor Positioning and Indoor Navigation, (Guimaraes, Portugal), Sep 2011.

[6] A. R. Jiménez, F. Seco, F. Zampella, and J. Prieto J.C.and Guevara, "Improved heuristic drift elimination with magnetic-aided dominant directions (mihde) for pedestrian navigation in complex buildings," Journal of Location Based Services, vol. 6, no. 3, pp. 186-210, 2012.

[7] C.-I. Chesneau, M. Hillion, and C. Prieur, "Motion estimation of a rigid body with an EKF using magneto-inertial measurements," in 2016 International Conference on Indoor Positioning and Indoor Navigation (IPIN), (Alcalá de Henares, Spain), Oct 2016.

[8] E. Dorveaux, Magneto-inertial navigation: principles and application to an indoor pedometer. PhD thesis, Ecole Nationale Supérieure des Mines de Paris, 2011.

[9] P. G. Savage, Strapdown Analytics by Paul G. Savage. Strapdown Associates, 2000.

[10] R. Sameni, "A linear Kalman notch filter for power-line interference cancellation," in 16th CSI International Symposium on Artificial Intelligence and Signal Processing, (Shirez, Iran), pp. 604-610, 2012.

[11] M. H. Afzal, V. Renaudin, and G. Lachapelle, "Magnetic field based heading estimation for pedestrian navigation environments," in 2011 International Conference on Indoor Positioning and Indoor Navigation, (Guimaraes, Portugal), Sep 2011.

[12] Widyawan, M. Klepal, and S. Beauregard, "A backtracking particle filter for fusing building plans with pdr displacement estimates," 2008 5th Workshop on Positioning, Navigation and Communication, Mar 2008.

[13] S. Beauregard, Widyawan, and M. Klepal, "Indoor PDR performance enhancement using minimal map information and particle filters," 2008 IEEE/ION Position, Location and Navigation Symposium, 2008.

[14] C. Ascher, C. Kessler, R. Weis, and G. Trommer, "Multi-floor map matching in indoor environments for mobile platforms," in 2012 International Conference on Indoor Positioning and Indoor Navigation (IPIN), (Sydney, Australia), Nov 2012. 